\title{
Coordinated Multi-Robot Exploration using a Segmentation of the Environment
}

\author{
Kai M. Wurm \\ Cyrill Stachniss \\ Wolfram Burgard
}

\begin{abstract}
This paper addresses the problem of exploring an unknown environment with a team of mobile robots. The key issue in coordinated multi-robot exploration is how to assign target locations to the individual robots such that the overall mission time is minimized. In this paper, we propose a novel approach to distribute the robots over the environment that takes into account the structure of the environment. To achieve this, it partitions the space into segments, for example, corresponding to individual rooms. Instead of only considering frontiers between unknown and explored areas as target locations, we send the robots to the individual segments with the task to explore the corresponding area. Our approach has been implemented and tested in simulation as well as in real world experiments. The experiments demonstrate that the overall exploration time can be significantly reduced by considering our segmentation method.
\end{abstract}

\section{INTRODUCTION}

Autonomous robots that are designed to create a map of their environment require the capability to effectively cover the space. There are several applications in which robots have been designed to autonomously explore their environment such as planetary exploration or in disaster missions. Using a coordinated team of robots instead of a single robot has often been suggested to be advantageous [4], [7] and cooperating robots have the potential to accomplish a task faster than a single robot [11]. By using several robots, redundancy can be explicitely introduced so that such a team can be expected to be more fault-tolerant than a single robot. Another advantage of robot teams arises from merging overlapping sensor information, which can help to compensate for sensor uncertainty. However, when robots operate in teams there is the risk of interference between them [10], [20]. For example, if the robots have the same type of active sensors such as ultrasound sensors, the overall performance can be reduced due to cross-talk. The more robots are used, the more time each robot may spend on detours in order to avoid collisions with other members of the team.

In this paper, we consider the problem of efficient exploration with teams of mobile robots that seek to minimize the overall time required to complete the mission. The entire task of coordinating a team of robots during exploration can roughly be separated into two subsequent tasks. First, one needs to identify potential exploration targets for the robots. Second, one needs to assign the individual robots to the target locations calculated in the previous step.

All authors are with the University of Freiburg, Department of Computer Science, D-79110 Freiburg, Germany

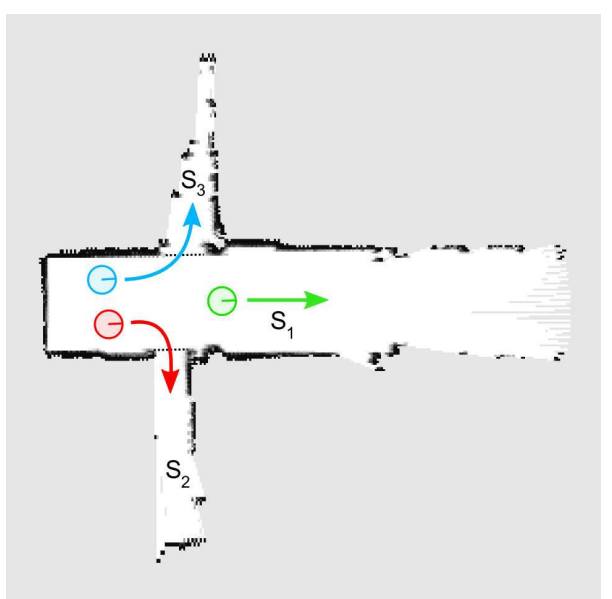

Fig. 1. Typical coordination of robots obtained by assigning them to different segments of the partial map.

A popular method for generating potential exploration targets has been proposed by Yamauchi et al. [26]. In this approach, robots are sent to so-called frontiers, which are given as the borders between the explored and the unexplored space. In a multi-robot context, it is important to carefully assign robots to targets so that redundant work and interference between robots is minimized. Therefore, it is the exploration strategy which affects the efficiency of the robot team the most. In many approaches, the robots are assigned directly to frontier targets based on a cost function that takes into account the expected path costs or travel time as well as a utility function that covers aspects such as the expected gain in information [3], [9], [21], [24], [28].

The coordination strategies described there consider individual locations rather than segments of the environment. Segmentation approaches which have recently received an increased amount of attention [2], [8], [23], [27] have originally been designed to facilitate topological localization and loop closing or have been used to reduce planning costs. In this paper, we introduce a new online coordination strategy for multi-robot exploration. It uses a segmentation of the already explored area to assign robots to segments instead of directly assigning them to frontier targets. Based on this segmentation, the robots are distributed over the environment more effectively which leads to a reduction of redundant work and the avoidance of interference between robots. As a result, the exploration time is significantly reduced. 
This paper is organized as follows. After discussing related work, we describe the Hungarian Method for target assignment in Section III. In Section IV, we introduce a graph-based method for map segmentation while in Section $\mathrm{V}$ we present our coordination approach. Finally, we describe simulated and real world experiments conducted to evaluate our approach.

\section{RELATED WORK}

The problem of exploring unknown terrains with teams of mobile robots has received considerable attention in the past. Yamauchi [26] presented a technique to learn maps with a team of mobile robots. He introduced the concept of frontiers between known and unknown areas in a grid map, which are widely used to select potential target locations during exploration. In this paper, we also consider frontiers but additionally utilize the structure of the environment for defining potential target locations. Koenig et al. [14] analyze different terrain coverage methods for small robots with limited sensing and computational capabilities. Furthermore, there has been research on how to deal with limited communication in the context of multi-robot exploration [3], [19].

An approach towards cooperation in heterogeneous robot systems has been presented by Singh and Fujimura [21]. If a robot is too big to pass through a narrow passage, it informs other robots about this task. Howard et al. [12] presented an incremental deployment approach that explicitly deals with obstructions, i.e., situations in which the path of one robot is blocked by another. Zlot and colleagues [28] proposed an architecture for mobile robot teams in which the exploration is guided by a market economy. They consider sequences of potential target locations for each robot and trade tasks between the robots using single-item first-price sealed-bid auctions. Such auction-based techniques have also been applied by Gerkey and Matarić [9] to efficiently solve the task allocation problem with a group of robots.

Matarić and Sukhatme [17] consider different strategies for task allocation in robot teams and analyze the performance of the team in extensive experiments. Ko et al. [13] present an approach that uses the Hungarian method [15] to compute the assignments of frontier cells to robots. In contrast to our work, Ko et al. mainly focus on finding a common frame of reference in case the start locations of the robots are not known.

In a previous work [24], we considered the problem of integrating semantic background information into the coordination procedure. This technique is related to the method proposed in this paper, even if the methodology is substantially different. Compared to our previous approach [24], we obtain a significantly reduced exploration time also for small teams of robot. The map segmentation technique used throughout this work is related to the spatial semantic hierarchy introduced by Kuipers and Byun [16]. The difference lies in the fact that we do not learn a model based on distinct places but utilize this information for a better coordination. Learning topological maps is itself a research field on its own and different methods have been proposed [2], [8], [25], [27]. These approaches are related to the technique described in this paper as they can be applied to separate the environment into appropriate regions that are then assigned to the individual robots.

\section{TARGET AssignMENT USING THE HUNGARIAN METHOD}

In 1955, Kuhn [15] presented a general method, which is often referred to as the Hungarian method, to assign a set of jobs to a set of machines given a fixed cost matrix. Consider a given $n \times n$ cost matrix which represents the cost of all individual assignments of jobs to machines. The Hungarian method, which is able to find the optimal solution with the minimal cost given this matrix, can be summarized by the following three steps:

1) Compute a reduced cost matrix by subtracting from each element the minimal element in its row. Afterwards, do the same with the minimal element in each column.

2) Find the minimal number of horizontal and vertical lines required to cover all zeros in the matrix. In case exactly $n$ lines are required, the optimal assignment is given by the zeros covered by the $n$ lines. Otherwise, continue with Step 3.

3) Find the smallest nonzero element in the reduced cost matrix that is not covered by a horizontal or vertical line. Subtract this value from each uncovered element in the matrix. Furthermore, add this value to each element in the reduced cost matrix that is covered by a horizontal and a vertical line. Continue with Step 2.

The computationally difficult part lies in finding the minimum number of lines covering the zero elements (Step 2). The overall algorithm has a complexity of $O\left(n^{3}\right)$. The method described above can directly be applied to assign a set of target locations (tasks) to the individual robots (machines). Here, each entry in the cost matrix can be the length of the path the corresponding robot has to travel to reach the designated target point.

Since the implementation of the Hungarian method described above requires the number of jobs and the number of machines to be equal, we need to slightly adapt the cost matrix computed in that way. This can be achieved by expanding the cost matrix using "dummy machines" (which will result in target locations that are not approached by any of the robots) and by duplicating existing targets. The Hungarian Method is then able to compute the optimal assignment, given the cost matrix.

\section{Map Segmentation}

Several researchers investigated the problem of segmenting maps based on the partitioning of a graph [1], [8], [16], [25], [27]. A very popular graph-based representation in this context are Voronoi Graphs (VGs) [5]. To compute the Voronoi Graph $G(m)=(V, E)$ of a given map $m$, we consider the set $O_{p}(m)$ which contain for each point $p$ in the free-space $C$ of $m$ the set of closest obstacle points. The 
Voronoi Graph then is given by the set of points in $O_{p}(m)$ for which there are at least two obstacle points with an equal minimal distance:

$$
\begin{aligned}
& V=\left\{p \in C|| O_{p}(m) \mid \geq 2\right\} \\
& E=\{(p, q) \mid p, q \in V, p \text { adjacent } q \text { in } m\}
\end{aligned}
$$

For each pair of nodes in $G(m)$ we add an edge if their corresponding points in $m$ are adjacent. The Voronoi Graph can be generated from metric maps of the environment such as occupancy grid maps [6], [25]. In a practical implementation this can be efficiently done by applying the Euclidean distance transformation [18] to an occupancy grid map. This transformation results in a distance map which holds for each grid cell the distance to the closest obstacle. A Voronoi Graph can then be constructed using skeletonization on the distance map. Figure 2 illustrates the process of generating a Voronoi Graph for an example occupancy grid map.

After generating the Voronoi Graph we are now interested in creating a partitioning of the graph into $k$ disjoint sets $V_{1}, V_{2}, \ldots, V_{k}$ with

$$
V=\bigcup_{i=1}^{k} V_{i}
$$

such that each cluster of nodes $V_{i}$ corresponds to a segment we can assign robots to. Thrun et al. suggest the graph to be separated at so-called critical points [25]. Here, critical points are those nodes in the Voronoi Graph at which the distance to the closest obstacle in the map is a local minimum. This is usually the case in doorways or other narrow passages.

Whereas this approach is able to reliably find doorways, it also generates a lot of false positive candidates in cluttered environments. To eliminate these false positives, we constrain them in the following way: First, critical points have to be nodes of degree 2 (two edges) and second, need to have a neighbor of degree 3 (a junction node). In addition, we require the points to lead from known into unknown areas, since segments which do not contain unknown areas can safely be ignored in an exploration task. To verify this constraint, we compute the distance to the closest reachable unknown cell for each point. This can be done efficiently in a similar way as the computation of the distance map. Figure 3 shows a pruned version of the Voronoi graph and the critical points found by our algorithm. All doorways have been selected as candidates and the number of false positives is much smaller than the number of critical points according to the definition of Thrun et al. [25] which includes distance minima in the Euclidean distance transformation within corridors and rooms.

In the practical experiments described in this paper we found that this segmentation technique yields sufficient results and allows to nicely distribute the robots. In unmodified office environments, we can typically reliably separate rooms and segments of a corridor. Other, more complex environments may however suggest more sophisticated segmentation algorithms which rely on hand-labeled training data [2], [8] or more complex reasoning [1], [27].
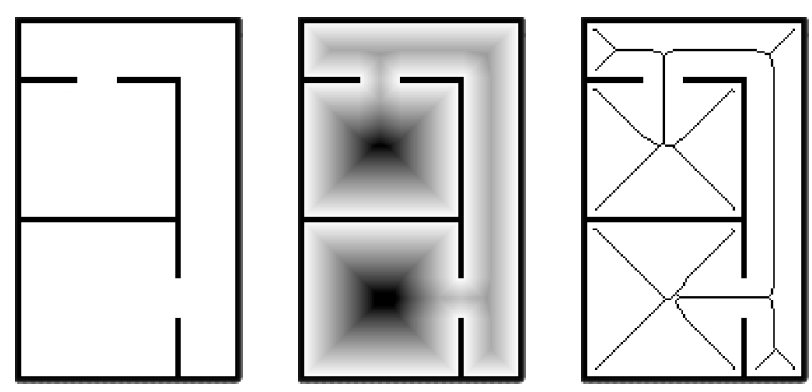

Fig. 2. Generation of the Voronoi Graph. Left: Example grid-map. Center: Map plus distance transform (the darker a point the larger the distance to the closest obstacle). Right: Map and Voronoi Graph generated from the distance transform using skeletonization.

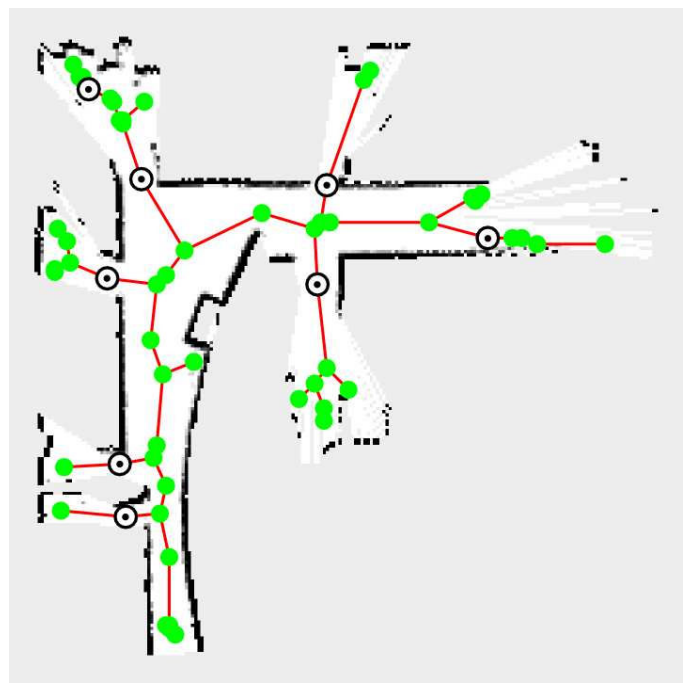

Fig. 3. Example segmentation of a small fraction of an environment. The marked nodes are the candidates for the partitioning of the graph calculated by our approach.

\section{Assignment of Robots to Target Areas}

Typical approaches to coordinated exploration seek to minimize the time needed to cover the whole environment with the robot's sensors. Therefore, it is often sub-optimal to explore the same (local) area with more than one robot. A cluster of robots which has a serious overlap in the field of view of the robots' sensors does not exploit its full potential. In practice, it is generally much more efficient to explore separate regions of the environment instead. For this reason, it is important to assign robots to exploration targets such that the robots do not get too close to each other during exploration.

Indoor environments are in general structured environments. Buildings are usually divided into rooms which can be reached via corridors. In many cases, it can be a disadvantage to assign more than one robot to one room. The room might, for example, be too small for a second robot to speed up it's exploration even though there initially is more than one frontier in the room. When the room is fully explored, robots might even block each other while trying to leave the room which will result in an increase in exploration time. 
In our approach, we assign individual robots to different segments of unexplored space. Segments could be separate rooms, corridors, or parts of larger corridors or rooms. This takes into account the structure of the environment and prevents the forming of inefficient clusters of robots.

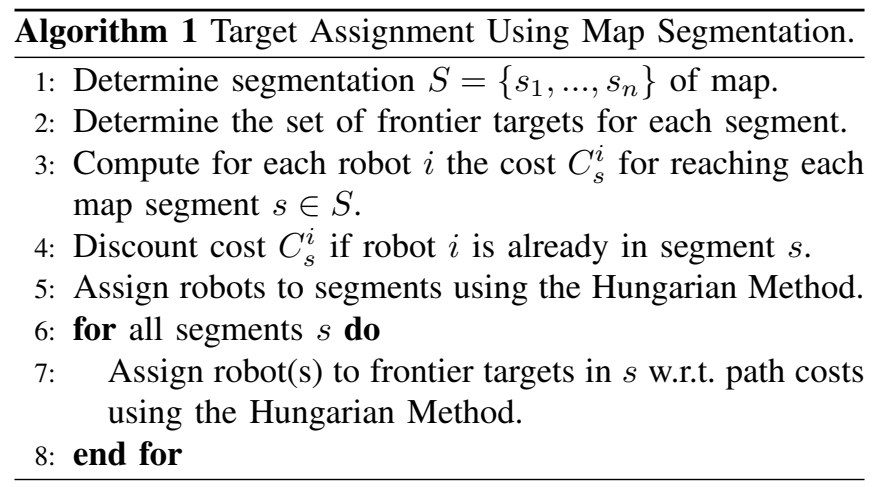

Our assignment algorithm is summarized in Algorithm 1. An assignment is determined whenever one of the robots requests a new exploration target. First, a partition of the partial map of the environment is created using the graphbased method described in Section IV. To generate targets within the segments, we then determine the set of frontier cells. The cost $C_{s}^{i}$ for reaching segment $s$ with robot $i$ is defined as the expected path cost to the nearest frontier cell within $s$. This cost is discounted by a constant factor if robot $i$ is already located in segment $s$. This has the effect that the robots stay in their assigned segment until it is completely explored. After computing the costs of a segment, an assignment is calculated by applying the Hungarian method (see Section III) based on the cost matrix.

The Hungarian method does not assign more than one robot to the same segment unless there are more robots available than there are unexplored segments. To appropriately handle those cases in which multiple robots are assigned to a single segment, we apply a local assignment based on the cost-optimal frontier within a segment. For this reason, our algorithm is equivalent to a purely frontier-based assignment if the environment cannot be partitioned, i.e., there is only one segment.

By assigning robots to separate segments, an appropriate distribution of the robots can be achieved. As we will demonstrate in the experiments, this leads to a significant reduction in exploration time. Instead of aiming at the closest frontier, robots share work more efficiently. A typical office environment, for example, contains corridors and rooms. Using our approach, each of the corridors is explored completely by one of the robots. In this way, the rough structure of the building will quickly be revealed. Meanwhile other robots will be assigned to the rooms reachable from the corridors, one at a time. This behavior does not only appear to be a natural way of exploring an unknown environment, our experiments also revealed that it significantly increases the efficiency of the robot team compared to approaches which ignore the structure of the building.

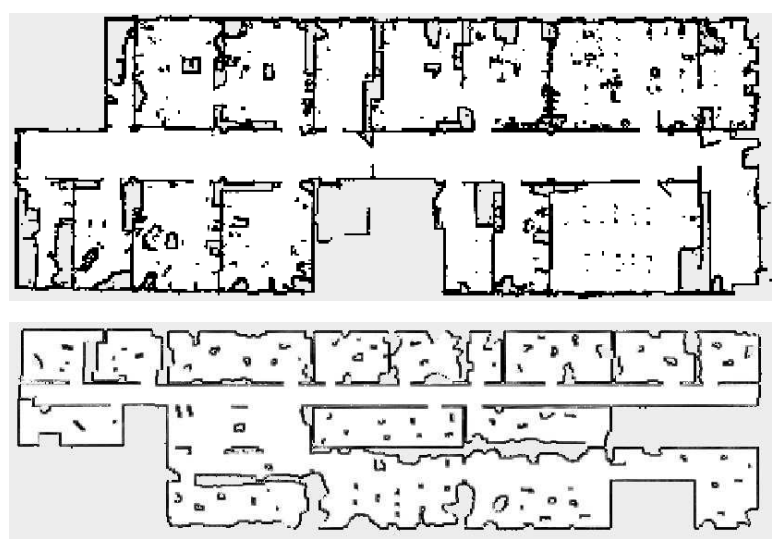

Fig. 4. Maps used in our simulated experiments: Building 079 of the Freiburg University (top) and Bremen University Cartesium (bottom).

Note that our algorithm is not limited to homogenous teams of robots. Consider the situation in which one particular robot cannot enter a certain part of the environment while another robot can. The assignment algorithm described above can be applied in this case by using modified segment costs $\bar{C}_{s}^{i}$ defined as:

$$
\begin{aligned}
\bar{C}_{s}^{i}= & \begin{cases}C_{s}^{i}, & , \text { if robot } i \text { can enter segment } s \\
\infty & , \text { otherwise. }\end{cases} \\
& \text { VI. EXPERIMENTAL RESULTS }
\end{aligned}
$$

\section{EXPERIMENTAL RESUlts}

Our approach has been implemented and evaluated using simulated as well as with real teams of robots. The real world experiments were conducted using two ActivMedia Pioneer II robots equipped with a laser range finder with a 180 degrees field of view. For generating the simulation results, we used the Carnegie Mellon Robot Navigation Toolkit. In all our experiments we assumed that the robots share a joint occupancy grid map, which is generated based on the sensor readings of all robots and under the assumption that all positions of the vehicles are known. This map is used for coordination, path planning, and path execution. We also assume that there is a central planning component which can communicate with all robot and can assign exploration targets to them. If there is only a limited communication range, then clusters of robots can be coordinated if one selects one individual planning agent per cluster [13], [22]. The experiments have been designed to verify that our exploration approach leads to significantly shorter exploration time compared to a standard frontier-based approach.

\section{A. Simulation Results}

To evaluate our robot coordination algorithm, we simulated teams of robots in various environments. We compared our segmentation-based approach to a frontier-based approach in which each robot is assigned to the closest frontier which has not been assigned to another robot yet. Since this strategy does not consider the structure of the environment, it will in general also assign more than one robot to one room or corridor if they contain more than one frontier. 
To eliminate influences from the segmentation algorithm used in the real world experiment, we assumed a given segmentation of the environment into rooms and corridors in our simulation experiments. As mentioned above, such a segmentation could also be reliably generated from the partial map alone.

Figure 4 depicts two maps of real environments used for the simulation (see also real world experiments). Both of them are office environments, one at the University of Freiburg and the other at the University of Bremen. To make the maps more different, we added clutter to the map representing the office environment located at the University of Bremen.

We varied the size of the simulated team from two to six robots (Freiburg map) respectively from two to eight robots (Bremen map). Since the Bremen map is considerably bigger than the Freiburg map, we simulated larger teams of robots there. For each team size, we conducted a series of simulated exploration runs starting from 20 different starting positions.

The results of our experiments can be seen in Figure 5. We measured the runtime gain of our approach which uses the assignment described in Section V compared to the alternative assignment described above. We plotted the runtime gain in percent of the total runtime against the size of the robot team. The error bars in the plots indicate the 95\% confidence level. It can be seen that our approach significantly outperforms the approach which does not use a segmentation based assignment.

The runtime gain is bigger for the Cartesium map since this map features several large rooms. This observation can be seen as an indicator as to when our approach will lead to especially good results. Whenever the environment can be divided into reasonably large and separated segments, our technique substantially reduces the overall exploration time.

In general, our strategy assigns one robot to one segment. As soon as there are more robots than segments multiple robots may be assigned to the same segment as mentioned in Section V. For this reason, the runtime gain of our strategy will decrease for large teams of robots in small environments. This can be seen in Figure 5. Note however, that the overall time to complete the mission still gets smaller the more robots are added to the task (the plot only shows the improvement of our approach vs. the frontier-based approach).

\section{B. Real Robot Experiments}

Our coordination algorithm has been evaluated using a team of real robots. For this experiment, we used two identical Pioneer II robots equipped with a laser range finder and a standard laptop-computer. During the experiment both robots were connected via a wireless network. The robot localization was achieved using a standard scan-matching approach. The relative starting poses of the robot were manually set in the beginning. Figure 6 depicts the two robots during their exploration mission.

The experiments were conducted in the lower floor of building 079 of the Freiburg computer science campus. The
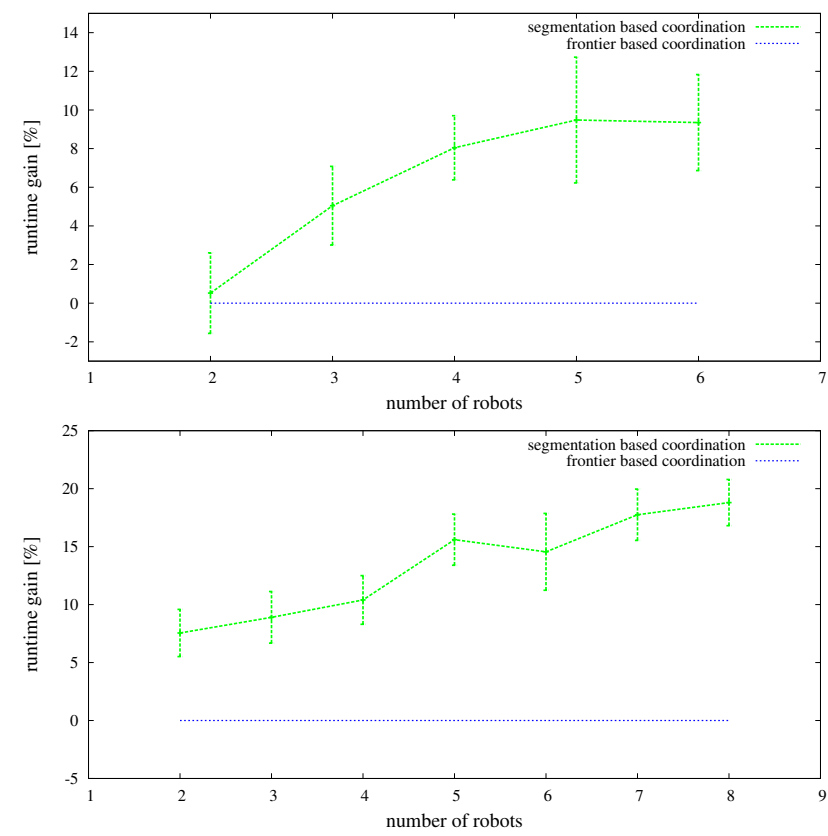

Fig. 5. Exploration time gain of our approach compared to a frontierbased approach for the AIS lab in Freiburg (top) and the Bremen Cartesium (bottom).

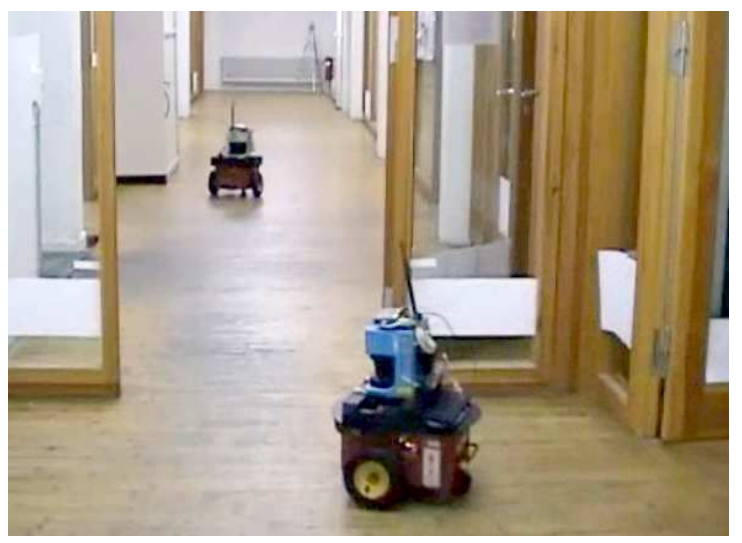

Fig. 6. Two robots exploring the AIS laboratory of the University of Freiburg using our coordination approach.

building has a size of approximately $37 \mathrm{~m} \times 14 \mathrm{~m}$ and consists of numerous office rooms and two long corridors divided by a door.

The team of robots was able to successfully explore the environment using our coordination approach. The result of one of the experiments can be seen in Figure 7. The figure shows the combined map of both robots after the exploration had finished. It also shows the trajectories of both robots during the exploration. The total exploration time was less than nine minutes, each of the robots traveled approximately $120 \mathrm{~m}$.

It can be seen that each of the rooms was explored by exactly one of the robots. It can also be seen that both corridors have been explored completely by one of the robots while the other one was exploring rooms reachable from the corridor. Another interesting effect is that the robots did not 


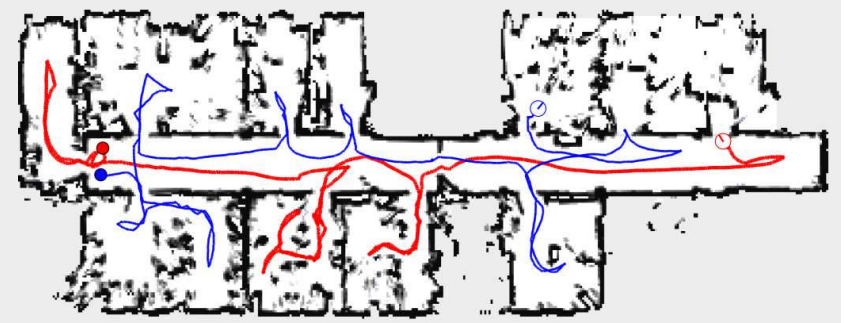

Fig. 7. Resulting map of the real world experiment including the trajectories of the two individual robots.

block each other during the execution of their tasks.

\section{CONCLUSION}

In this paper, we proposed a novel technique for coordinating a team of exploring robots. We use a segmentation of the environment to determine exploration targets for the individual robots. By assigning each robot to a separate segment, a balanced distribution of the robots over the environment is achieved. This leads to a shorter overall exploration time compared to an approach which does not use our segmentation. Thus, our approach reduces the risk of interference between robots and the amount of redundant work. We also introduced an efficient graph-based segmentation technique for partially explored environments. Our approach has been implemented and evaluated in simulation as well as with a team of real robots. The experiments show a significant improvement of the segmentation-based approach compared to a standard frontier-based approach for structured indoor environments. Note that our approach is not limited to our segmentation method. Using a heterogenous team of robots, for example, such a segmentation can be defined based on traversability constraints of the different robots.

\section{ACKNOWLEDGMENT}

This work has partly been supported by the German Research Foundation (DFG) under contract number SFB/TR8 (A3).

\section{REFERENCES}

[1] P. Beeson, N.K. Jong, and B. Kuipers. Towards autonomous topological place detection using the extended voronoi graph. In Proc. of the IEEE Int. Conf. on Robotics \& Automation (ICRA), 2005.

[2] E. Brunskill, T. Kollar, and N. Roy. Topological mapping using spectral clustering and classification. In Proc. of the IEEE/RSJ Int. Conf. on Intelligent Robots and Systems (IROS), San Diego, October 2007.

[3] W. Burgard, M. Moors, C. Stachniss, and F. Schneider. Coordinated multi-robot exploration. IEEE Transactions on Robotics, 21(3):376378, 2005.

[4] Y.U. Cao, A.S. Fukunaga, and A.B. Khang. Cooperative mobile robotics: Antecedents and directions. Journal of Autonomous Robots, 4(1):7-27, 1997.

[5] H. Choset, , and Burdick J. Sensor-based exploration: The hierarchical generalized voronoi graph. J. of Robotics Research, 19(2), 2000.

[6] H. Choset and J. Burdick. Sensor based planning, part i: The generalized voronoi graph. In Proc. of the IEEE Int. Conf. on Robotics \& Automation (ICRA), Nagoya, Japan, 1995.

[7] G. Dudek, M.. Jenkin, E. Milios, and D. Wilkes. A taxonomy for multi-agent robotics. Journal of Autonomous Robots, 3(4):375-397, 1996.
[8] S. Friedman, H. Pasula, and D. Fox. Voronoi random fields: Extracting topological structure of indoor environments via place labeling. In Manuela M. Veloso, editor, Proc. of the Int. Conf. on Artificial Intelligence (IJCAI), pages 2109-2114, 2007.

[9] B.P. Gerkey and M.J. Matarić. Sold!: Auction methods for multirobot coordination. IEEE Transactions on Robotics and Automation, 18(5):758- 768, 2002.

[10] D. Goldberg and M.J. Matarić. Interference as a tool for designing and evaluating multi-robot controllers. Journal of Robotics \& Autonomous Systems, 8:637-642, 1997.

[11] D. Guzzoni, A. Cheyer, L. Julia, and K. Konolige. Many robots make short work. AI Magazine, 18(1):55-64, 1997.

[12] A. Howard, M.J. Matarić, and S. Sukhatme. An incremental deployment algorithm for mobile robot teams. In Proc. of the IEEE/RSJ Int. Conf. on Intelligent Robots and Systems (IROS), pages 2849-2854, Lausanne, Switzerland, 2002.

[13] J. Ko, B. Stewart, D. Fox, K. Konolige, and B. Limketkai. A practical, decision-theoretic approach to multi-robot mapping and exploration. In Proc. of the IEEE/RSJ Int. Conf. on Intelligent Robots and Systems (IROS), pages 3232-3238, Las Vegas, NV, USA, 2003.

[14] S. Koenig, B. Szymanski, and Y. Liu. Efficient and inefficient ant coverage methods. Annals of Mathematics and Artificial Intelligence, 31:41-76, 2001.

[15] H.W. Kuhn. The hungarian method for the assignment problem. Naval Research Logistics Quarterly, 2(1):83-97, 1955.

[16] B. Kuipers and Y.-T. Byun. A robot exploration and mapping strategy based on a semantic hierarchy of spatial representations. Journal of Robotics \& Autonomous Systems, 8:47-63, 1991.

[17] M.J. Matarić and G. Sukhatme. Task-allocation and coordination of multiple robots for planetary exploration. In Proc. of the Int. Conf. on Advanced Robotics (ICAR), pages 61-70, Budapest, Hungary, 2001.

[18] A. Meijster, J.B.T.M. Roerdink, and W.H. Hesselink. Mathematical Morphology and its Applications to Image and Signal Processing, chapter A General Algorithm for Computing Distance Transforms in Linear Time, pages 331-340. Kluwer Academic Publishers, 2000.

[19] I. Rekleitis, V. Lee-Shue, A. Peng New, and H. Choset. Limited communication, multi-robot team based coverage. In Proc. of the IEEE Int. Conf. on Robotics \& Automation (ICRA), pages 3462-3468, New Orleans, LA, USA, 2004.

[20] M. Schneider-Fontan and M.J. Matarić. Territorial multi-robot task division. IEEE Transactions on Robotics and Automation, 14(5):815822, 1998.

[21] K. Singh and K. Fujimura. Map making by cooperating mobile robots. In Proc. of the IEEE Int. Conf. on Robotics \& Automation (ICRA), pages 254-259, Atlanta, GA, USA, 1993.

[22] C. Stachniss. Exploration and Mapping with Mobile Robots. $\mathrm{PhD}$ thesis, University of Freiburg, Department of Computer Science, April 2006.

[23] C. Stachniss, G. Grisetti, O. Martínez-Mozos, and W. Burgard. Efficiently learning metric and topological maps with autonomous service robots. it - Information Technology, 49(4):232-238, 2007.

[24] C. Stachniss, O. Martínez-Mozos, and W. Burgard. Speeding-up multi-robot exploration by considering semantic place information. In Proc. of the IEEE Int. Conf. on Robotics \& Automation (ICRA), pages 1692-1697, Orlando, FL, USA, 2006.

[25] S. Thrun. Learning metric-topological maps for indoor mobile robot navigation. Artificial Intelligence, 99(1):21-71, 1998.

[26] B. Yamauchi. Frontier-based exploration using multiple robots. In Proc. of the Second International Conference on Autonomous Agents, pages 47-53, Minneapolis, MN, USA, 1998.

[27] Z. Zivkovic, B. Bakker, and B. Kröse. Hierarchical map building and planning based on graph partitioning. In Proc. of the IEEE Int. Conf. on Robotics \& Automation (ICRA), pages 803-809, 2006.

[28] R. Zlot, A.T. Stenz, M.B. Dias, and S. Thayer. Multi-robot exploration controlled by a market economy. In Proc. of the IEEE Int. Conf. on Robotics \& Automation (ICRA), Washington, DC, USA, 2002. 